\title{
ИНТЕРИКТАЛЬНАЯ ЭПИЛЕПТИФОРМНАЯ АКТИВНОСТЬ ВО СНЕ И В БОДРСТВОВАНИИ У ПАЦИЕНТОВ С ВИСОЧНОЙ ЭПИЛЕПСИЕЙ
}

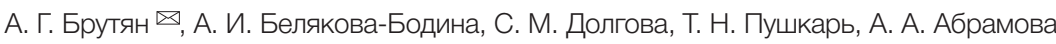 \\ Научный центр неврологии, Москва, Россия
}

\begin{abstract}
Сон является значимым активатором эпилептиформной активности, при этом вероятность регистрации разрядов может меняться в зависимости от стадии и глубины сна. Целью исследования было оценить зависимость эпилептиформной активности от уровня бодрствования и глубины сна в популяции взрослых пациентов с височной локализацией разрядов. Нами было проанализировано 32 продолженных ЭЭГ-мониторинга с записью ночного сна, в которых были отмечены все фокальные разряды, а также проводилось стадирование сна. Определяли общий индекс эпилептиформной активности, как отношение общего количества разрядов к длительности исследования в часах, а также индивидуальные индексы эпилептиформной активности для бодрствования и каждой из стадий сна: для REM-сазы и N1, N2 и N3 стадий NREM-сна. У подавляющего числа пациентов (28) максимальный индекс зарегистрирован в стадии N3. По мере перехода к более глубоким стадиям сна, индекс разрядов последовательно и достоверно увеличивался, достигая максимальных величин в стадии N3 ( $p<0,01)$. В фазу REM-сна индекс резко снижался $(p<0,01)$, приближаясь к значениям, зарегистрированным в бодрствовании. Основным механизмом, который приводит к увеличению индекса эпилептиформной активности в NREM-сне, может быть нарастающая синхронизация корковых нейронов с увеличением доли медленной активности в ЭЭГ. Таким образом, стадия 스 является наиболее информативной в плане регистрации фокальных разрядов, что диктует необходимость достижения достаточной глубины сна при проведении диагностических ЭЭГ исследований у пациентов с височными формами эпилепсии
\end{abstract}

Ключевые слова: электроэнцефалография, сон, эпилептиформная активность, эпилепсия, височная эпилепсия

Информация о вкладе авторов: А. Г. Брутян - планирование исследования, анализ литературы, интерпретация данных, подготовка черновика рукописи; А. И. Белякова-Бодина, А. А. Абрамова - сбор, анализ, интерпретация данных, подготовка черновика рукописи; С. М. Долгова, Т. Н. Пушкарь сбор, анализ данных.

Соблюдение этических стандартов: исследование одобрено этическим комитетом ФГБНУ НЦН (протокол № 1-4/19 от 23 января 2019 г.)

$\bowtie$ Для корреспонденции: Амаяк Грачевич Брутян

Ленинградский проспект, д. 33, г. Москва, 125284; abroutian@mail.ru

Статья получена: 23.08.2019 Статья принята к печати: 30.09.2019 Опубликована онлайн: 02.11.2019

DOI: 10.24075 /vrgmu.2019.073

\section{INTERICTAL EPILEPTIFORM ACTIVITY IN SLEEP AND WAKEFULNESS IN PATIENTS WITH TEMPORAL LOBE EPILEPSY}

Broutian AG $\bowtie$, Belyakova-Bodina Al, Dolgova SM, Pushkar TN, Abramova AA

Research Center of Neurology, Moscow, Russia

\begin{abstract}
Sleep is an important activator of epileptiform activity, with epileptiform discharge (ED) probability varying among sleep stages. The aim of our study was to analyze the association between epileptiform activity and sleep stages or wakefulness in adults with temporal discharges. We analyzed 32 long-term overnight EEG recordings. All focal discharges were marked, and the entire sleep was staged. Absolute general epileptiform discharge index (EDI), defined as a ratio of total ED number to the full recording time in hours, as well as absolute EDIs for REM, N1, N2 and N3 stages were calculated. The majority of patients (28) had the highest EDI in N3. EDI increased significantly while sleep progressed to deeper stages, reaching its peak in N3. In REM sleep, EDI sharply declined $(p<0.01)$ reaching the levels of wakefulness. Increasing synchronization of cortical neurons is thought to be the major mechanism of EDI rise in NREM sleep. Hence, N3 seems to be the most sensitive stage to capture EDs, which highlights the importance of deep sleep recording in patients
\end{abstract} with temporal epilepsy.

Keywords: electroencephalography, sleep, epileptiform activity, epilepsy, temporal lobe epilepsy

Author contribution: Broutian AG — research planning, literature analysis, data interpretation, manuscript drafting; Belyakova-Bodina Al, Abramova AA — data collection, analysis, interpretation, manuscript drafting; Dolgova SM, Pushkar TN — data collection, analysis.

Compliance with ethical standards: the study was approved by the Research Center of Neurology Ethics Committee (protocol № 1-4/19 of January 23, 2019).

$\triangle$ Correspondence should be addressed: Amayak G. Broutian

Leningradsky Prospect, 33, Moscow, 125284; abroutian@mail.ru

Received: 23.08.2019 Accepted: 30.09.2019 Published online: 02.11.2019

DOI: $10.24075 /$ brsmu.2019.073

Вероятность развития эпилептических приступов в зависимости от уровня бодрствования и глубины сна в значительной степени определяют форма эпилепсии и тип приступов [1, 2]. Например, для юношеской миоклонической эпилепсии характерны приступы после пробуждения. При аутосомно-доминантной ночной лобной эпилепсии приступы возникают исключительно во сне. Известно также, что сон служит значимым активатором интериктальной (межприступной) эпилептиформной активности в электроэнцефалографии (ЭЭГ), причем для фокальных разрядов в большей степени, чем для генерализованных [3]. Сон, однако, не является однородным состоянием, а представляет собой довольно цикличную смену нескольких состояний, которые отличаются глубиной, физиологическими показателями и паттернами ЭЭГ. Наиболее ярким примером служит эпилептическая энцефалопатия с продолженной спайкволновой активностью во сне (continuous spikes and waves during sleep, CSWS), при которой эпилептические разряды практически полностью замещают физиологические паттерны NREM-сна (non-rapid eye movements sleep, сон без «быстрых движений глаз»), однако регрессируют в 
фазу REM (rapid eye movements) сна. Таким образом, следует ожидать различную вероятность регистрации эпилептиформной активности в ЭЭГ в зависимости от стадии сна. Представляется целесообразным не только учитывать абсолютное количество разрядов в той или иной стадии, но и соотносить их с общей продолжительностью стадий за время сна. Так, в норме наибольшее общее время приходится на 2-ю стадию, поэтому неудивительно, что может создаваться ошибочное впечатление о большей вероятности регистрации разрядов во второй стадии.

Индексы эпилептиформной активности описаны как при генерализованных, так и при фокальных формах эпилепсии [4-8]. Опубликованные работы по фокальным разрядам различаются количеством пациентов, локализацией разрядов, методами подсчета индексов эпилептиформной активности. В значительной части исследований разряды подсчитывали выборочно - на фрагментах стадий сна и бодрствования, от 5 до 20 мин в каждой стадии. Как локализация очага, так и методы расчета индекса разрядов могут оказывать влияние на результаты [4-8].

Височная локализация очага - наиболее частая, и, по некоторым данным, составляет около 2/3 от общего количества случаев локализационно-обусловленной эпилепсии [9]. Целью исследования было изучить влияние различных стадий сна на интериктальную эпилептиформную активность ЭЭГ на относительно однородной группе взрослых пациентов с височной эпилепсией с полным подсчетом разрядов на протяжении всей записи.

\section{ПАЦИЕНТЫ И МЕТОДЫ}

Из общего числа пациентов, которые прошли обследование в лаборатории видео-ЭЭГ мониторинга ФГБНУ НЦН в период с февраля 2018 г. по июнь 2019 г., из соображений стандартизации времени исследования были выбраны ночные видео-ЭЭГ мониторинги (НВЭМ) с включением вечернего и утреннего бодрствований длительностью 10-12 ч в количестве 709 исследований. Первоначально из этого числа отобрали 142 записи, в которых была зарегистрирована фокальная эпилептиформная активность у пациентов вне зависимости от длительности заболевания и приема противоэпилептических препаратов. Критерии включения: 1) наличие разрядов только височной локализации; 2) в случае повторных обращений пациентов для анализа использовали запись, выполненную при первом визите; 3) наличие регистрации всех стадий сна; 4) все разряды отмечены врачом вручную, без использования алгоритма автоматической детекции разрядов; 5) общее количество разрядов не менее 10. Критерии исключения: 1) записи с зарегистрированными эпилептическими приступами; 2) с количеством разрядов более 500. Записи с приступами исключали в связи с их потенциальным влиянием на индекс эпилептиформной активности. Записи с очень большим количеством разрядов (более 500) исключали, поскольку ручная маркировка эпилептиформной активности в таких случаях затруднительна. Окончательное количество пациентов, записи ЭЭГ которых соответствовали критериям и вошли в анализ, составило 32, из них 17 женщин и 15 мужчин, в возрасте от 19-79 лет (медиана 41 год).

Запись ЭЭГ производили скальповыми электродами по международной системе 10-20 и электрокардиографическим
(ЭКГ) каналом, в 11 из 32 записей использовали дополнительные электроды “Скуловой дуги» (F9, F10, T9, T10, P9, Р10). Для регистрации ЭЭГ использовали электроэнцефалографы моделей NicOne (Natus; CШA) и BePlus LTM (EBNeuro; Италия). Исследование начинали в 20:00-21:00 и завершали в 7:00-8:00 ч. В начале записи проводили пробы с ритмической фотостимуляцией и 5-минутной гипервентиляцией, которые повторяли после утреннего пробуждения. Эпилептиформную активность определяли в соответствии с пересмотренным глоссарием рекомендованных терминов ЭЭГ от 2017 г. [10]. Количество разрядов на данной выборке пациентов зарегистрировали в диапазоне от 13 до 401 за время записи (медиана - 72 разряда за ночь). У 27 пациентов (84\%) разряды регистрировались только с одной стороны, у 5 пациентов - битемпорально. Количество пациентов с локализацией (или преобладанием) разрядов в левой височной области (17) было сопоставимо с количеством пациентов с исключительно или преимущественно правосторонней локализацией (15).

Стадирование сна и бодрствования осуществляли на 30-секундных эпохах в соответствии с рекомендациями Американской ассоциации медицины сна [11]. Поскольку при записи не использовали электроокулографические (ЭОГ) каналы и электромиографические (ЭМГ) каналь мышц подбородка, стадирование REM-сна осуществляли косвенно на основании глазодвигательных артефактов в лобных отделах и миографических артефактов в каналах ЭЭГ, а также наличия ЭЭГ-потенциалов, характерных для REM-сна [11]. В данной публикации использованы традиционные обозначения для стадий сна и бодрствования: Wake - бодрствование, N1 - первая стадия сна, N2 - вторая стадия сна, N3 - третья стадия сна (медленноволновой сон), REM - REM-сон. На рис. 1 представлен пример гипнограммы с отметками разрядов.

Для каждого из исследований после построения гипнограммы и разметки разрядов вначале проводили расчет следующих показателей: 1) общего индекса эпилептиформной активности, определяемого как отношение общего числа разрядов ко всей длительности записи в часах; 2) индексов эпилептиформной активности для бодрствования и каждой стадии сна (N1, N2, N3, REM), определяемых как отношение числа разрядов в стадии ко всему времени стадии за время записи в часах. В случае битемпоральных разрядов рассчитывали объединенный средний индекс разрядов с обеих сторон. Был отмечен довольно большой разброс полученных абсолютных значений (индексов). Поскольку в данном исследовании нас интересовали прежде всего тренды индексов в зависимости от стадии, проводили дополнительную нормализацию: рассчитывали относительный индекс каждой стадии (отношение индекса разрядов каждой стадии к общему индексу разрядов). В дальнейшем тексте под индексом разрядов, если не указано иное, следует понимать именно относительные индексы эпилептисормной активности для бодрствования и каждой стадии сна.

Статистическую обработку результатов проводили с применением программы STATISTICA 12.6 (Statsoft, Dell Software; США). Нормальность распределения признаков проверяли методом Шапиро-Уилка, использовали методы непараметрической статистики: Манна-Уитни для независимых выборок и критерий Уилкоксона для зависимых выборок. Корреляцию рассчитывали по Спирмену. Различия считали статистически значимыми при $p<0,05$. 


\section{РЕЗУЛЬТАТЫ ИССЛЕДОВАНИЯ}

В стадии бодрствования разряды встречались в 18 случаях из 32 (56\%), в стадии N1 у 16 (50\%), в N2 - у 31 из 32 (97\%), в стадии N3 - у всех 32 (100\%), в REM-фазу у 9 пациентов (28\%) (табл. 1). Отмечены различные сочетания стадий сна, в которых была зарегистрирована эпилептиформная активность (рис. 2). Не было ни одного пациента, у которого разряды регистрировали исключительно в бодрствовании, N1, N2 или REM; в одном случае разряды были зарегистрированы исключительно в стадии N3.

Были подсчитаны стадии с максимальным количеством индекса разрядов за исследование. Наибольшее количество - 28 (87,5\%) записей пришлось на стадию дельта-сна N3, в 2 случаях при бодрствовании, у 1 - в N2, еще у 1 - в REM, ни у одного пациента в нашей выборке не было максимума в стадии N1.

Данные относительных индексов в бодрствовании и по всем стадиям сна с трендами по всем пациентам представлены на рис. З. Были получены следующие групповые значения по стадиям (медиана): 0,03 при бодрствовании, 0,11 - в стадии N1, 0,19 - в стадии N2, 3,92 - в N3 и 0,19 - в фазу REM-сна (табл. 1, рис. 4). Статистический анализ (парный критерий Уилкоксона) показал, что по сравнению с бодрствованием индекс разрядов в NREM-сне достоверно выше: $p<0,05$ для стадии N1 и $p<0,01$ для стадий N2 и N3, разница с фазой REM незначима. Значения индекса в N2 выше, чем в N1 и REM $(p<0,01)$. Относительный индекс в стадии N3 достоверно превышает значения во всех остальных стадиях $(p<0,01)$. Индекс в REM-фазе достоверно уступает всем стадиям NREM-сна и, как указано ранее в тексте, слабо отличается от индекса в бодрствовании.

Вся выборка была разделена на 2 группы: с низким (от 14, минимального в выборке количества разрядов за исследование, до 75) и относительно высоким (от 75 до максимального в выборке значения, равного 401) количеством разрядов. Разница в индексах разрядов между группами с низким и высоким количеством разрядов была статистически недостоверной.

Проведен корреляционный анализ между показателями абсолютных индексов разрядов при бодрствовании и в различных стадиях сна между собой. Обнаружена статистически значимая корреляция между следующими парами: положительные корреляции индекса в бодрствовании с индексом в N1 и REM, N1 с N2, а также N2 с N3.

Достоверной разницы индексов эпилептиформной активности в бодрствовании и во сне между левой и правой стороной височных разрядов не обнаружено.

\section{ОБСУЖДЕНИЕ РЕЗУЛЬТАТОВ}

По результатам нашего исследования, индексы в стадии дельта-сна значительно превышают таковые в остальных стадиях сна и бодрствовании. Максимальная представленность разрядов в фазу медленноволнового сна показана в предыдущих работах, в которых индекс эпилептиформной активности анализировали по отдельным стадиям сна $[6-8,12-14]$. В нашем исследовании среди пациентов с фокальными разрядами не было ни одного, у кого разряды не регистрировались бы в стадии N3, при этом вероятность их регистрации превышала в среднем в 3 раза таковую в стадии N2. Еще большая разница проявляется при сопоставлении стадий с максимальным количеством разрядов, где количество записей с максимумом разрядов в N3 составило 87,5\%. При анализе численных значений (табл. 1) и графика трендов индексов (рис. 3) отмечали всплеск в стадии N3, а также схожую динамику индекса разрядов в стадии N2, значительно уступающего численно N3 и превышающего значения в бодрствовании, стадии N1 и фазе REM-сна. Достоверное различие индексов эпилептиформной активности в стадиях N1 и N2 у пациентов с фокальной эпилептиформной активностью было показано нами впервые. Вероятно, отсутствие достоверных различий между этими стадиями в предыдущих исследованиях можно связать с применявшейся в данных работах

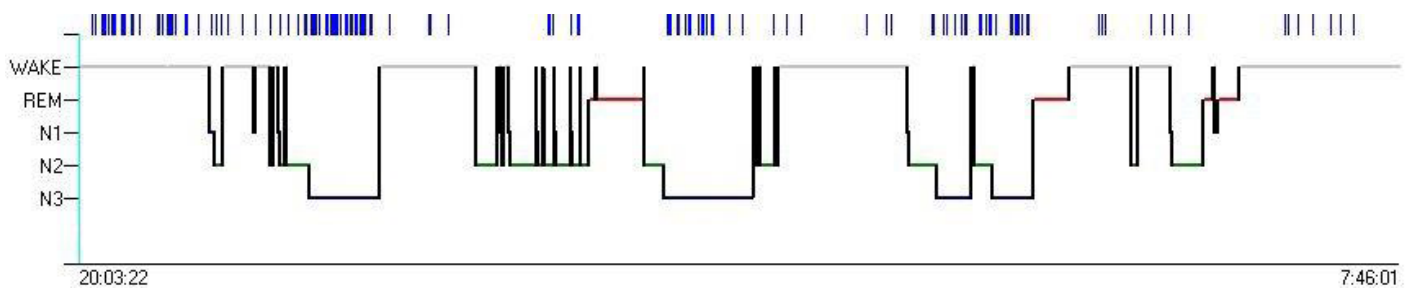

Рис. 1. Пример гипнограммы с разметкой разрядов. На оси абсцисс отложено астрономическое время. Вертикальные линии над гипнограммой отметки о разрядах эпилептиформной активности. Wake - бодрствование, N1 - первая стадия сна, N2 - вторая стадия сна, N3 - третья стадия сна (медленноволновой сон), REM - REM-сOH

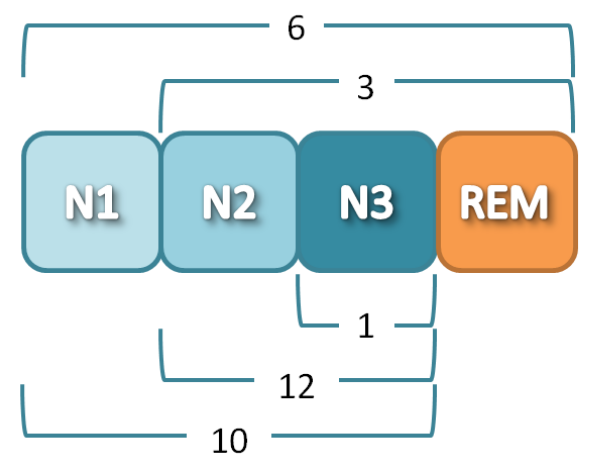

Рис. 2. Зарегистрированные комбинации встречаемости эпилептиформной активности в различных стадиях сна. Фигурными скобками выделень характерные сочетания стадий сна, в которых была зарегистрирована эпилептиформная активность. Значение над фигурной скобкой - число пациентов с данным распределением разрядов 
Таблица 1. Количество записей с разрядами и средние значения индексов эпилептиформной активности по стадиям сна и бодрствования, нормированные в отношении общего индекса разрядов за исследование

\begin{tabular}{|l|c|c|c|c|}
\hline \multicolumn{1}{|c|}{ Стадия } & Wake & N1 & N2 & REM \\
\hline $\begin{array}{l}\text { Количество пациентов с разрядами/без разрядов } \\
\text { в данной стадии }\end{array}$ & $18 / 14$ & $16 / 16$ & $31 / 1$ & $32 / 0$ \\
\hline $\begin{array}{l}\text { Количество пациентов с максимальным числом разрядов } \\
\text { в указанной стадии }\end{array}$ & 2 & 0 & 1 \\
\hline $\begin{array}{l}\text { Относительный индекс разрядов, медиана } \\
\text { [нижний; верхний квартиль] }\end{array}$ & $0,03[0 ; 0,23]$ & $0,11[0 ; 0,89]$ & $1,19[0,64 ; 1,64]$ & $3,92[2,74 ; 6,64]$ \\
\hline
\end{tabular}

выборочной разметкой эпилептифформной активности $[8,15,16]$.

Таким образом, выявлено возрастание индекса эпилептиформной активности по мере увеличения глубины сна от REM-фазы к стадии N1, N2 и N3.

Рассмотрим вероятный физиологический механизм, объясняющий найденные закономерности изменения индекса разрядов. Известно, что для регистрации потенциалов в скальповой ЭЭГ необходима синхронная активность достаточно большого количества корковых нейронов. По экспериментальным данным, площадь таких синхронно работающих нейронов должна составлять не менее 6-8 см². Медленная активность, в первую очередь дельта-диапазона, является отражением большой степени синхронизации корковых нейронов. Критерием стадии N3 является наличие не менее 20\% высокоамплитудных дельта-колебаний в эпохе. Однако при тщательном анализе можно видеть, что постепенное нарастание медленной активности отмечается уже во второй стадии.
Помимо ритмической медленноволновой активности, не достигающей, однако, критериев 3-й стадии, в N2 регистрируются К-комплексы, являющиеся ее важнейшим критерием и фактически представляющие собой отдельные высокоамплитудные относительно медленные одиночные потенциалы, в которые нередко встраиваются разряды. Это так называемые эпилептизированные К-комплексы, которые регистрируются как при генерализованных, так и при фокальных эпилепсиях [17-18]. Более того, имеются данные, указывающие на возможную связь приступов при ночной лобной эпилепсии с К-комплексами [19]. Таким образом, можно рассматривать высокую вероятность регистрации разрядов в NREM-сне как следствие нарастания синхронизации работы нейронных сетей, что также подтверждают работы, в которых проводили прямую корреляцию частоты разрядов с мощностью дельта-колебаний [20].

Несмотря на то что сон в целом является сильнейшим активатором эпилептиформной активности, в REM-фразе

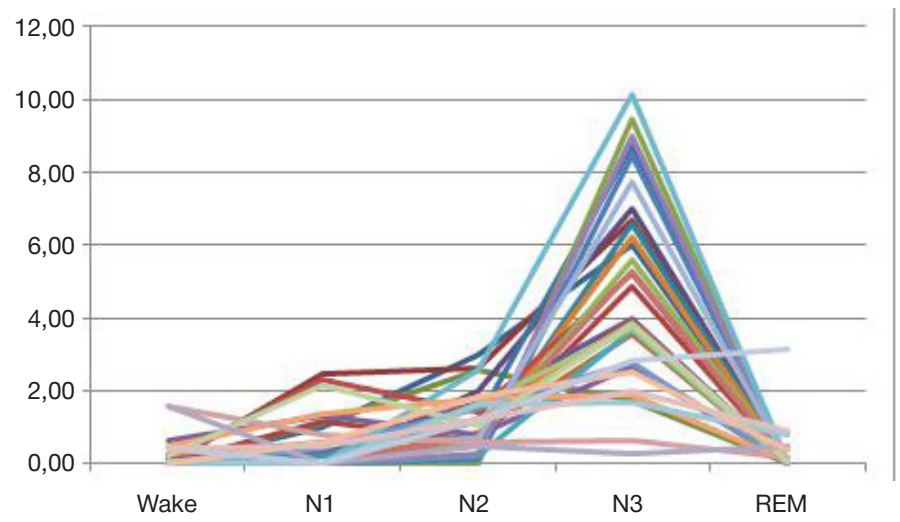

Рис. 3. Тренды индивидуальных индексов эпилептиформной активности при бодрствовании и по стадиям сна у всех пациентов с височной локализацией эпилептиформной активности

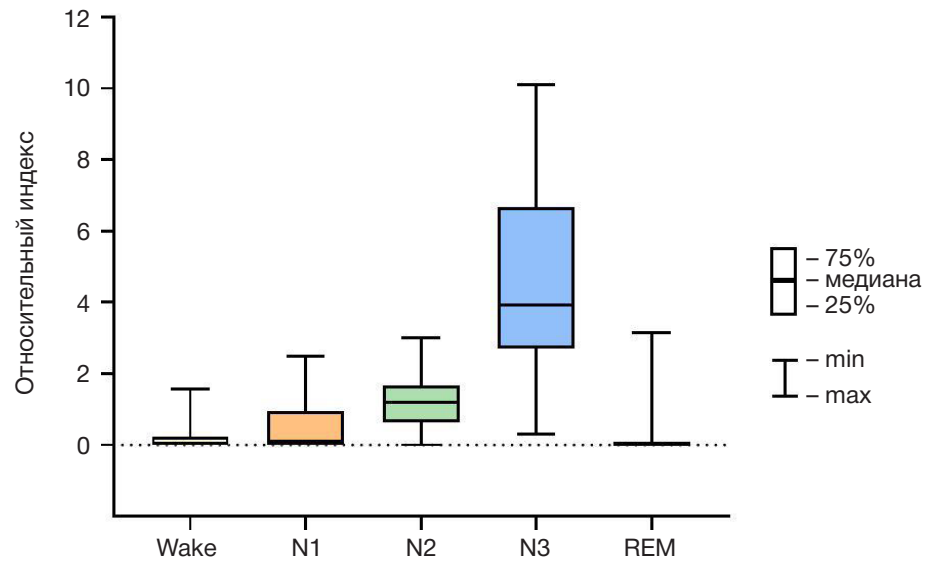

Рис. 4. Значения относительного индекса эпилептиформной активности по стадиям сна и бодрствования у всех пациентов с височной локализацией эпилептиформной активности 
представленность разрядов была наиболее низкой, сопоставимой с таковой при бодрствовании. Наши данные согласуются с предыдущими работами, в которых исследовали влияние REM-сна на эпилептиформную активность [8, 14, 21-22]. Во всех исследованиях индекс в REM-фразу был наименьшим во сне. Если сравнивать с бодрствованием, то в большинстве работ индекс разрядов при бодрствовании был несколько выше. Не являясь активатором эпилептиформной активности, REMсон, однако, привлекает особое внимание в двух аспектах. Во-первых, если разряды в REM все-таки регистрируются, они носят более фокальный характер и имеют, таким образом, более высокое локализационное значение, чем другие стадии сна [23-24]. Во-вторых, REM, являясь неотьемлемой частью структуры здорового сна, не просто не потенцирует разряды, но и снижает их вероятность. Таким образом, функционирование нейронных сетей в REM носит в какой-то степени протективный характер, снижая вероятность не только разрядов, но и приступов [3].

Следует отметить, что на результаты нашего исследования могли повлиять критерии включения пациентов, в частности, мы сознательно исключили пациентов с очень большим количеством разрядов, которые практически невозможно подсчитать вручную. В некоторых предыдущих исследованиях $[15,8,14,25]$ применяли выборочный метод подсчета разрядов, основанный на регистрации разрядов на фрагментах стадий (5-20 мин, 1 ч) с экстраполяцией на все исследование. Мы также применяем выборочную разметку у пациентов с большим количеством разрядов, однако решили не включать эти записи в настоящее исследование, предполагая, что они могут потенциально сказаться на результатах. Кроме того, поскольку мы не использовали ЭМГ-регистрацию (m. mentalis), это могло привести к некоторой недооценке продолжительности REM-сна. Значения индексов, которые мы получили для бодрствования, могли также оказаться несколько завышенными, поскольку в бодрствование вошли и 5-минутные пробы с гипервентиляцией, которая, снижая мозговой кровоток, увеличивает степень синхронизации нейронов и является потенциальным активатором эпилептиформной активности. Однако NREM является существенно более сильным активатором эпилептисоорной активности, чем гипервентиляция [15]. В данной работе не оценивали также возможное влияние противоэпилептической терапии, времени от дебюта приступов, частоты приступов, их семиологии, наличия фармакорезистентного течения и структурной патологии на МРТ.

С практической точки зрения результаты нашего исследования подчеркивают необходимость регистрации медленноволнового сна в диагностических исследованиях у пациентов с подозрением на фокальную форму эпилепсии. Короткие записи, в частности рутинная ЭЭГ бодрствования, продолжительность которой не превышает 20 мин, недостаточно чувствительны в диагностике эпилепсии, прежде всего ее фокальных форм, что может приводить к задержке в постановке точного диагноза и назначения соответствующего лечения в связи с отсутствием на таких записях достаточной глубины сна.

\section{ВЫВОДЫ}

Результаты настоящего исследования на группе пациентов с височной локализацией эпилептиформной активности показывают возрастание индекса фокальных разрядов по мере увеличения глубины NREM-сна от первой к третьей стадии. B фазе REM-сна индекс значительно снижается до уровня, сопоставимого с бодрствованием. Наиболее вероятным механизмом достоверного преобладания разрядов в стадии медленноволнового сна представляется синхронизация работы корковых нейронов. Отсутствие стадии N3, в которой представленность эпилептиформной активности максимальна, или недостаточная продолжительность этой стадии значительно снижают вероятность регистрации эпилептиформной активности, что в случае недостаточно убедительных клинических проявлений может приводить к ошибочным выводам, задержке в постановке правильного диагноза и начала терапии.

\section{Литература}

1. Minecan D, Natarajan A, Marzec M, Malow B. Relationship of epileptic seizures to sleep stage and sleep depth. Sleep. 2002; 25 (8): 899-904.

2. Bazil CW. Seizure modulation by sleep and sleep state. Brain Res. 2019; (1703): 13-17.

3. Ng M, Pavlova M. Why are seizures rare in rapid eye movement sleep? Review of the frequency of seizures in different sleep stages. Epilepsy Res Treat. 2013; (2013): 932790.

4. Kellaway P, Frost JD, Crawley JW. Time modulation of spikeand-wave activity in generalized epilepsy. Ann Neurol. 1980; 8 (5): 491-500.

5. Halász P, Terzano MG, Parrino L. Spike-wave discharge and the microstructure of sleep-wake continuum in idiopathic generalised epilepsy. Neurophysiol Clin. 2002; 32 (1): 38-53.

6. Lieb JP, Joseph JP, Engel J, Walker J, Crandall PH. Sleep state and seizure foci related to depth spike activity in patients with temporal lobe epilepsy. Electroencephalogr Clin Neurophysiol. 1980; 49 (5-6): 538-57.

7. Sammaritano M, Gigli GL, Gotman J. Interictal spiking during wakefulness and sleep and the localization of foci in temporal lobe epilepsy. Neurology. 1991; 41 (2 (Pt 1): 290-7.

8. Clemens Z, Janszky J, Clemens B, Szucs A, Halász P. Factors affecting spiking related to sleep and wake states in temporal lobe

epilepsy (TLE). Seizure. 2005; 14 (1): 52-7.

9. Semah F, Picot MC, Adam C, Broglin D, Arzimanoglou A, Bazin B, et al. Is the underlying cause of epilepsy a major prognostic factor for recurrence? Neurology. 1998; 51 (5): 1256-62.

10. Kane N, Acharya J, Benickzy S, Caboclo L, Finnigan S, Kaplan PW, et al. A revised glossary of terms most commonly used by clinical electroencephalographers and updated proposal for the report format of the EEG findings. Revision 2017. Clin Neurophysiol Pract. 2017; (2): 170-85.

11. Berry RB, Brooks R, Gamaldo CE, Harding SM, Lloyd RM, Marcus CL, et al. The AASM Manual for the Scoring of Sleep and Associated Events: Rules, Terminology and Technical Specifications, Version 2.2. www.aasmnet.org. Darien, Illinois: American Academy of Sleep Medicine, 2015.

12. Malow BA, Kushwaha R, Lin X, Morton KJ, Aldrich MS. Relationship of interictal epileptiform discharges to sleep depth in partial epilepsy. Electroencephalogr Clin Neurophysiol. 1997; 102 (1): 20-6.

13. Clemens Z, Janszky J, Szucs A, Békésy M, Clemens B, Halász P. Interictal epileptic spiking during sleep and wakefulness in mesial temporal lobe epilepsy: a comparative study of scalp and foramen ovale electrodes. Epilepsia. 2003; 44 (2): 186-92.

14. Singh S, Shukla G, Goyal V, Srivastava AK, Singh MB, Vibha D, et al. Impact of sleep on the localizing value of video EEG in patients 
with refractory focal seizures - a prospective video-EEG with EOG and submental EMG study. Clin Neurophysiol. 2014; 125 (12): 2337-43.

15. Klein KM, Knake S, Hamer HM, Ziegler A, Oertel WH, Rosenow F. Sleep but not hyperventilation increases the sensitivity of the EEG in patients with temporal lobe epilepsy. Epilepsy Res. 2003; 56 (1): 43-9.

16. Yu YL, Yan YL, Tian SF, Feng ZH, Shi MT. Circadian rhythm of interictal epileptiform discharges and changes of spindles in patients with temporal lobe epilepsy. Biomed Res. 2018; 29 (6): 1263-7.

17. Geyer JD, Carney PR, Gilliam F. Focal epileptiform spikes in conjuction with K-complexes. J Clin Neurophysiol. 2006; 23 (5): 437-40.

18. Niedermeyer E. Epileptiform $\mathrm{K}$ complexes. Am J Electroneurodiagnostic Technol. 2008; 48 (1): 48-51.

19. ElHelou J, Navarro V, Depienne C, Fedirko E, LeGuern E, Baulac M, et al. K-complex-induced seizures in autosomal dominant nocturna frontal lobe epilepsy. Clin Neurophysiol. 2008; 119 (10): 2201-4.

20. Ferrillo F, Beelke M, DeCarli F, Cossu M, Munari C, Rosadini G, et al. Sleep-EEG modulation of interictal epileptiform discharges in

\section{References}

1. Minecan D, Natarajan A, Marzec M, Malow B. Relationship of epileptic seizures to sleep stage and sleep depth. Sleep. 2002; 25 (8): 899-904

2. Bazil CW. Seizure modulation by sleep and sleep state. Brain Res. 2019; (1703): 13-17.

3. $\mathrm{Ng} \mathrm{M}$, Pavlova M. Why are seizures rare in rapid eye movement sleep? Review of the frequency of seizures in different sleep stages. Epilepsy Res Treat. 2013; (2013): 932790.

4. Kellaway P, Frost JD, Crawley JW. Time modulation of spikeand-wave activity in generalized epilepsy. Ann Neurol. 1980; 8 (5): 491-500.

5. Halász P, Terzano MG, Parrino L. Spike-wave discharge and the microstructure of sleep-wake continuum in idiopathic generalised epilepsy. Neurophysiol Clin. 2002; 32 (1): 38-53.

6. Lieb JP, Joseph JP, Engel J, Walker J, Crandall PH. Sleep state and seizure foci related to depth spike activity in patients with temporal lobe epilepsy. Electroencephalogr Clin Neurophysiol. 1980; 49 (5-6): 538-57.

7. Sammaritano M, Gigli GL, Gotman J. Interictal spiking during wakefulness and sleep and the localization of foci in temporal lobe epilepsy. Neurology. 1991; 41 (2 (Pt 1): 290-7.

8. Clemens Z, Janszky J, Clemens B, Szucs A, Halász P. Factors affecting spiking related to sleep and wake states in temporal lobe epilepsy (TLE). Seizure. 2005; 14 (1): 52-7.

9. Semah F, Picot MC, Adam C, Broglin D, Arzimanoglou A, Bazin B, et al. Is the underlying cause of epilepsy a major prognostic factor for recurrence? Neurology. 1998; 51 (5): 1256-62.

10. Kane N, Acharya J, Benickzy S, Caboclo L, Finnigan S, Kaplan PW, et al. A revised glossary of terms most commonly used by clinica electroencephalographers and updated proposal for the repor format of the EEG findings. Revision 2017. Clin Neurophysiol Pract. 2017; (2): 170-85

11. Berry RB, Brooks R, Gamaldo CE, Harding SM, Lloyd RM, Marcus CL, et al. The AASM Manual for the Scoring of Sleep and Associated Events: Rules, Terminology and Technical Specifications, Version 2.2. www.aasmnet.org. Darien, Illinois: American Academy of Sleep Medicine, 2015.

12. Malow BA, Kushwaha R, Lin X, Morton KJ, Aldrich MS. Relationship of interictal epileptiform discharges to sleep depth in partial epilepsy. Electroencephalogr Clin Neurophysiol. 1997; 102 (1): 20-6.

13. Clemens Z, Janszky J, Szucs A, Békésy M, Clemens B, Halász P. Interictal epileptic spiking during sleep and wakefulness in mesial temporal lobe epilepsy: a comparative study of scalp and foramen ovale electrodes. Epilepsia. 2003; 44 (2): 186-92. adult partial epilepsy: a spectral analysis study. Clin Neurophysiol. 2000; 111 (5): 916-23.

21. Malow BA, Lin X, Kushwaha R, Aldrich MS. Interictal spiking increases with sleep depth in temporal lobe epilepsy. Epilepsia. 1998; 39 (12): 1309-16.

22. Campana C, Zubler F, Gibbs S, deCarli F, Proserpio P, Rubino A1, et al. Suppression of interictal spikes during phasic rapid eye movement sleep: a quantitative stereo-electroencephalography study. J Sleep Res. 2017; 26 (5): 606-13.

23. Malow BA, Aldrich MS. Localizing value of rapid eye movement sleep in temporal lobe epilepsy. Sleep Med. 2000; 1 (1): 57-60.

24. Ochi A, Hung R, Weiss S, Widjaja E, To T, Nawa Y, et al. Lateralized interictal epileptiform discharges during rapid eye movement sleep correlate with epileptogenic hemisphere in children with intractable epilepsy secondary to tuberous sclerosis complex. Epilepsia. 2011; 52 (11): 1986-94.

25. Scarlatelli-Lima AV, Sukys-Claudino L, Watanabe N, Guarnieri R, Walz R, Lin K. How do people with drug-resistant mesial temporal lobe epilepsy sleep? A clinical and video-EEG with EOG and submental EMG for sleep staging study. eNeurologicalSci. 2016; (4): 34-41

14. Singh S, Shukla G, Goyal V, Srivastava AK, Singh MB, Vibha D, et al. Impact of sleep on the localizing value of video EEG in patients with refractory focal seizures - a prospective video-EEG with EOG and submental EMG study. Clin Neurophysiol. 2014; 125 (12): 2337-43.

15. Klein KM, Knake S, Hamer HM, Ziegler A, Oertel WH, Rosenow F. Sleep but not hyperventilation increases the sensitivity of the EEG in patients with temporal lobe epilepsy. Epilepsy Res. 2003; 56 (1): 43-9.

16. Yu YL, Yan YL, Tian SF, Feng ZH, Shi MT. Circadian rhythm of interictal epileptiform discharges and changes of spindles in patients with temporal lobe epilepsy. Biomed Res. 2018; 29 (6): 1263-7.

17. Geyer JD, Carney PR, Gilliam F. Focal epileptiform spikes in conjuction with K-complexes. J Clin Neurophysiol. 2006; 23 (5): 437-40.

18. Niedermeyer E. Epileptiform $\mathrm{K}$ complexes. Am J Electroneurodiagnostic Technol. 2008; 48 (1): 48-51.

19. ElHelou J, Navarro V, Depienne C, Fedirko E, LeGuern E, Baulac M, et al. K-complex-induced seizures in autosomal dominant nocturnal frontal lobe epilepsy. Clin Neurophysiol. 2008; 119 (10): 2201-4.

20. Ferrillo F, Beelke M, DeCarli F, Cossu M, Munari C, Rosadini G, et al. Sleep-EEG modulation of interictal epileptiform discharges in adult partial epilepsy: a spectral analysis study. Clin Neurophysiol. 2000; 111 (5): 916-23.

21. Malow BA, Lin X, Kushwaha R, Aldrich MS. Interictal spiking increases with sleep depth in temporal lobe epilepsy. Epilepsia 1998; 39 (12): 1309-16.

22. Campana C, Zubler F, Gibbs S, deCarli F, Proserpio P, Rubino A1, et al. Suppression of interictal spikes during phasic rapid eye movement sleep: a quantitative stereo-electroencephalography study. J Sleep Res. 2017; 26 (5): 606-13.

23. Malow BA, Aldrich MS. Localizing value of rapid eye movement sleep in temporal lobe epilepsy. Sleep Med. 2000; 1 (1): 57-60.

24. Ochi A, Hung R, Weiss S, Widjaja E, To T, Nawa Y, et al. Lateralized interictal epileptiform discharges during rapid eye movement sleep correlate with epileptogenic hemisphere in children with intractable epilepsy secondary to tuberous sclerosis complex. Epilepsia. 2011; 52 (11): 1986-94.

25. Scarlatelli-Lima AV, Sukys-Claudino L, Watanabe N, Guarnieri R, Walz R, Lin K. How do people with drug-resistant mesial temporal lobe epilepsy sleep? A clinical and video-EEG with EOG and submental EMG for sleep staging study. eNeurologicalSci. 2016; (4): $34-41$ 